\title{
Detecting Shadow of Moving Object based on Phong Illumination Model
}

\author{
Fei Peng $\mathrm{Li}^{1,2, a}$, Zongxi Song ${ }^{1}$, Bin $\mathrm{Li}^{1,2}$, Meng Jie $\mathrm{Wu}^{1,2}$ and Chao Shen ${ }^{1,2}$ \\ ${ }^{1}$ Space Optics Laboratory, Xi'an Institute of Optics and Precision Mechanics of CAS, Xi'an, China \\ ${ }^{2}$ University of Chinese Academy of Sciences, Beijing, China \\ a1172391152@qq.com
}

Keywords: shadow detection; Phong illumination model; curve fitting; steepest descent direction.

\begin{abstract}
When detecting moving objects using visible light image sequence, the shadows of moving objects always appear together with moving objects. Shadows usually change with light condition, which brings difficulty for recognizing objects after segmenting image. This text proposes an algorithm to detect shadows in foreground image according to the Phong illumination model. The algorithm combines the brightness information between foreground image and background image to calculate an array of brightness relative variation ratio, detecting shadows using the statistical feature of shadows in the array. The algorithm uses the statistical histogram to describe statistical feature of brightness relative variation ratio, and the Gaussian model to do curve fitting as a description for the shape of statistical histogram. And finally with the property of the fitted function could we find the threshold value of shadows adaptively.
\end{abstract}

\section{Introduction}

When using popular algorithms to detect moving objects, among those algorithms such as optical flow [1], frame difference [2], background subtraction, usually a frame of image is segmented as foreground image and background image. In foreground image, moving objects and shadows of moving objects always appear together. Among the popular algorithms used for object recognition, shadows are obstacle for achieving accuracy of the feature extracted from objects of the algorithm. How to remove shadows accurately and completely become an important work before further recognition and tracking of moving objects. At present, there are three categories of algorithm to detect shadows, based on color, texture, and statistical information [3]. This text detects shadows based on the illumination features in foreground image according to Phong illumination model. Experiments prove that the algorithm has a good effect and less calculation.

\section{Analysis of Shadow Area Illumination Feature}

According to Phong illumination model [4], brightness reflected from objects mainly comes from ambient light, diffuse reflection light, specular reflection light. Assume I the brightness of object, $\mathrm{I}_{\mathrm{e}}$ the light intensity of ambient light, $\mathrm{I}_{\mathrm{s}}$ the light intensity of direct light from light source, $\mathrm{K}_{\mathrm{a}}, \mathrm{K}_{\mathrm{b}}, \mathrm{K}_{\mathrm{c}}$ are the reflection coefficients, $\theta$ is the included angle between incident light and the surface normal, $\alpha$ is the included angle between reflected light and gazing direction, $n_{s}$ is the coefficient of specular reflection, so the brightness of object could be defined as Eq. 1.

$$
\mathrm{I}=\mathrm{K}_{\mathrm{a}} \mathrm{I}_{\mathrm{e}}+\mathrm{K}_{\mathrm{b}} \mathrm{I}_{\mathrm{s}} \cos \cos \theta+\mathrm{K}_{\mathrm{c}} \mathrm{I}_{\mathrm{s}} \cos ^{\mathrm{n}_{\mathrm{s}}} \alpha .
$$

In real world scenarios, most objects surface are not smooth, hence assume the contribution of specular reflection light for brightness could be ignored, means $K_{c} I_{s} \cos ^{n_{s}} \alpha$ to be $0 . K_{a}$ and $K_{b}$ could be considered approximately as the same. Assume $\mathrm{I}^{\mathrm{F}}(\mathrm{x}, \mathrm{y})$ to be the brightness value of pixel point $(x, y)$ in foreground image, $I^{B}(x, y)$ to be the brightness value of pixel point $(x, y)$ in background image, define the brightness relative variation ratio to be as Eq. 2 . 


$$
\Delta \mathrm{I}=\frac{\mathrm{I}^{\mathrm{F}}(\mathrm{x}, \mathrm{y})}{\mathrm{I}^{\mathrm{B}}(\mathrm{x}, \mathrm{y})} .
$$

With the analysis above, combining Eq. 1 and Eq. 2 we have Eq. 3.

$$
\Delta \mathrm{I}=\frac{\mathrm{K}_{\mathrm{a}}^{\mathrm{F}} \mathrm{I}_{\mathrm{e}}^{\mathrm{F}}+\mathrm{K}_{\mathrm{b}}^{\mathrm{F}} \mathrm{I}_{\mathrm{s}}^{\mathrm{F}} \cos \cos \theta^{\mathrm{F}}}{\mathrm{K}_{\mathrm{a}}^{\mathrm{B}} \mathrm{I}_{\mathrm{e}}^{\mathrm{B}}+\mathrm{K}_{\mathrm{b}}^{\mathrm{B}} \mathrm{I}_{\mathrm{s}}^{\mathrm{B}} \cos \cos \theta^{\mathrm{B}}} .
$$

For $\mathrm{K}_{\mathrm{a}}^{\mathrm{F}}=\mathrm{K}_{\mathrm{b}}^{\mathrm{F}}, \mathrm{K}_{\mathrm{a}}^{\mathrm{B}}=\mathrm{K}_{\mathrm{b}}^{\mathrm{B}}$, make $\mathrm{K}_{\mathrm{a}}^{\mathrm{F}}=\mathrm{K}_{\mathrm{b}}^{\mathrm{F}}=\mathrm{K}^{\mathrm{F}}, \mathrm{K}_{\mathrm{a}}^{\mathrm{B}}=\mathrm{K}_{\mathrm{b}}^{\mathrm{B}}=\mathrm{K}^{\mathrm{B}}$, make $\mathrm{C}=\mathrm{K}^{\mathrm{F}} / \mathrm{K}^{\mathrm{B}}$, Eq. 3 can be simplified as Eq. 4.

$$
\Delta \mathrm{I}=\mathrm{C} \frac{\mathrm{I}_{\mathrm{e}}^{\mathrm{F}}+\mathrm{I}_{\mathrm{s}}^{\mathrm{F}} \cos \cos \theta^{\mathrm{F}}}{\mathrm{I}_{\mathrm{e}}^{\mathrm{B}}+\mathrm{I}_{\mathrm{s}}^{\mathrm{B}} \cos \cos \theta^{\mathrm{B}}} .
$$

As for shadow area, direct light from light source is kept out, $\mathrm{I}_{\mathrm{s}}^{\mathrm{F}}=0$, Eq. 4 turns to Eq. 5 .

$$
\Delta I_{s}=C \frac{I_{e}^{F}}{I_{e}^{B}+I_{s}^{B} \cos \cos \theta^{B}} .
$$

In both foreground and background image, the light intensity of ambient light is the same, thus $\mathrm{I}_{\mathrm{e}}^{\mathrm{F}}=\mathrm{I}_{\mathrm{e}}^{\mathrm{B}}$, assume light condition won't change dramatically, so $\mathrm{I}_{\mathrm{s}}^{\mathrm{B}} / \mathrm{I}_{\mathrm{e}}^{\mathrm{F}}$ can be considered as constant. Shadows actually are cast over background, thus $\mathrm{K}^{\mathrm{F}}=\mathrm{K}^{\mathrm{B}}$, and $\mathrm{C}=1 . \theta^{\mathrm{B}}$ is a constant value too. Based on analysis above, we can infer that the value of $\Delta \mathrm{I}_{\mathrm{s}}$ is relatively stable and most likely to concentrate in a small range. However, the brightness relative variation ratio of objects $\Delta \mathrm{I}_{\mathrm{o}}$ has a wider range of distribution.

\section{Shadow Detection}

Text [5] proposes two Equations to judge shadow point. One is to calculate covariance of all point in $\Delta \mathrm{I}$ array on a $5 \times 5$ sized template. The other is to filter $\Delta \mathrm{I}$ array with a special designed template. On the basis of analysis above, this article uses statistical histogram to decide the threshold value for shadow in $\Delta \mathrm{I}$ array. This text uses Gaussian Mixture Model to build background model and accomplish segmentation of foreground image and background image. Draw a statistical histogram to describe the statistical feature of $\Delta \mathrm{I}$ array. As shown in Fig. 1, the feature of shadows in $\Delta \mathrm{I}$ array can be concluded as follow: (1)For the value of $\Delta \mathrm{I}_{\mathrm{s}}$ at different point is more stable and may concentrate in a small range, hence, in statistical histogram there should be a peak which is always the highest and steepest. Of course zero does not count because zero represent background in foreground image; (2)Direct light is kept out in shadow area which is always dimmer, resulting in the highest peak reach before 85(ratio less than 1); (3)The value for shadow range from the first minimum value at the left side of the highest peak to the first minimum value at the right side of the highest peak. According to the features presented in statistical histogram of $\Delta \mathrm{I}$ array, decide a threshold interval $\left[\mathrm{T}_{\mathrm{l}}, \mathrm{T}_{\mathrm{r}}\right]$, judge shadow point as Eq. 6.

$$
\mathrm{P}(\mathrm{x}, \mathrm{y})\left\{\begin{array}{cc}
1 & \mathrm{~T}_{1}<\Delta \mathrm{I}(\mathrm{x}, \mathrm{y})<\mathrm{T}_{\mathrm{R}} \\
0 & \text { otherwise }
\end{array}\right. \text {. }
$$

Consider $(x, y)$ as shadow point where $P(x, y)=1$. Use the algorithm to process foreground image as Fig. 2, the result is shown in Fig. 3.

\section{Adaptive Threshold}

Using covariance or specific template to judge shadow point demands determine threshold value manually which lacking of flexibility and may cause ineffectiveness of algorithm. Consider describe the shape of histogram by curve fitting. And with the property of the fitted curve could we find the threshold value for shadow adaptively. Use different methods for curve fitting. Experiments show 
that smoothing spline and Gaussian have better smoothness than polynomial and interpolation. And in this specific application, smooth curve can better describe the trend of histogram. In this text, Gaussian model is used to do curve fitting. Through experiments do we find when using the sum of six or seven Gaussian function as the fitting model could we get a smooth and relatively accurate curve to describe histogram which is definitely beneficial for threshold decision at next stage. Gaussian model is presented as Eq. 7.

$$
\mathrm{f}(\mathrm{x})=\mathrm{a}_{1} \mathrm{e}^{\left[-\left(\frac{\mathrm{x}-\mathrm{b}_{1}}{\mathrm{c}_{1}}\right)^{2}\right]}+\mathrm{a}_{2} \mathrm{e}^{\left[-\left(\frac{\mathrm{x}-\mathrm{b}_{2}}{\mathrm{c}_{2}}\right)^{2}\right]}+\ldots+\mathrm{a}_{\mathrm{n}} \mathrm{e}^{\left[-\left(\frac{\mathrm{x}-\mathrm{b}_{\mathrm{n}}}{\mathrm{c}_{\mathrm{n}}}\right)^{2}\right]} .
$$

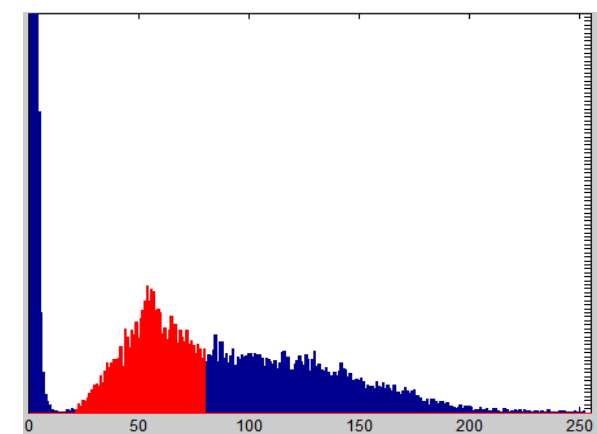

Fig. 1 histogram for $\Delta \mathrm{I}$

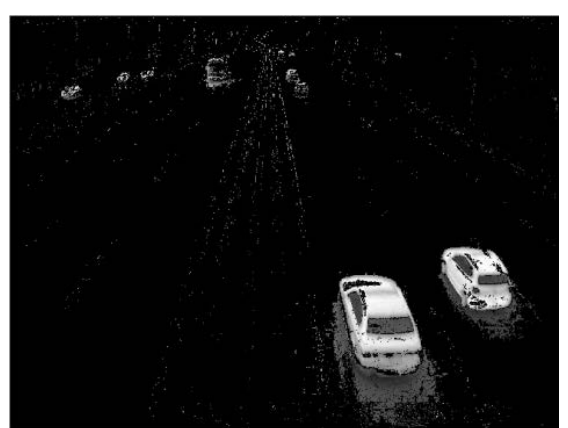

Fig. 2 foreground image

For Fig. 1, use Gaussian model that $\mathrm{n}=7$ to do curve fitting and result is shown in Fig. 4.

On the basis of fitting result could we determine the function expression for Gaussian model, defined as $\mathrm{g}(\mathrm{x})$. It is easy to find the maximum value and the point where the peak appears. Take the left and right neighbor point as initial points, search minimum points on both sides respectively using steepest descent direction. Calculation method is described as follow: (1)Assume initial point $\mathrm{x}^{1} \in \mathrm{R}^{\mathrm{n}}$, precision $\varepsilon>0$, make $k=0$. (2) Calculate $\nabla g\left(x^{k}\right)$, if $\nabla g\left(x^{k}\right) \leq \varepsilon$, stop, $x^{*} \approx x^{k}$; otherwise turn to (3). (3) $\mathrm{p}^{\mathrm{k}}=-\nabla \mathrm{g}\left(\mathrm{x}^{\mathrm{k}}\right)$. (4) Solve LS: $\min _{\gamma \geq 0} \min _{\gamma \geq 0} g\left(\mathrm{x}^{\mathrm{k}}+\gamma \mathrm{p}^{\mathrm{k}}\right)=\mathrm{g}\left(\mathrm{x}^{\mathrm{k}}+\gamma_{\mathrm{k}} \mathrm{p}^{\mathrm{k}}\right)$ to get $\gamma_{\mathrm{k}}$, make $\mathrm{x}^{\mathrm{k}+1}=\left(\mathrm{x}^{\mathrm{k}}+\gamma_{\mathrm{k}} \mathrm{p}^{\mathrm{k}}\right), \mathrm{k}=\mathrm{k}+1$, turn to (2). Use method above to find minimum $\mathrm{T}_{1}$ and $\mathrm{T}_{\mathrm{r}}$ to be threshold for shadows in $\Delta \mathrm{I}$ array.

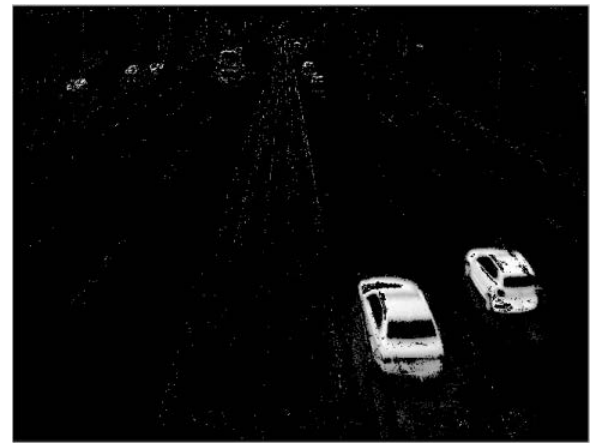

Fig. 3 foreground image after shadow removal

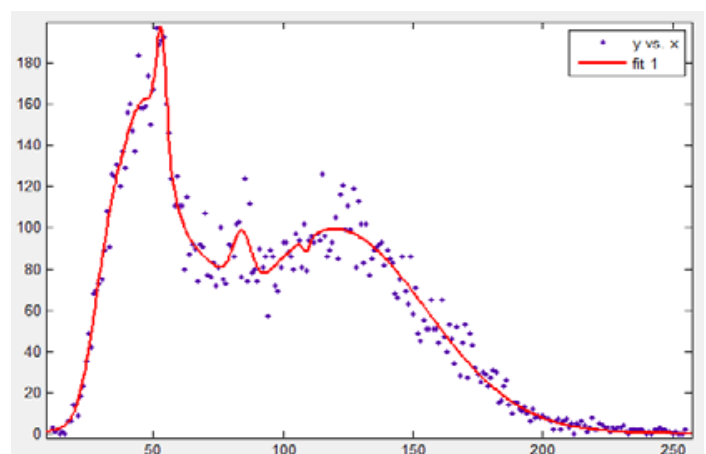

Fig. 4 curve fitting by Gaussian

\section{Results and Analysis}

There are two ways to test performance of the algorithm, quantitatively and qualitatively.

A shadow detection algorithm with good performance should avoid detecting shadow as object as well as detecting object as shadow. Define $\omega_{1}$ to be the probability of shadow being correctly detected, and $\omega_{2}$ to be the probability of object being correctly detected. Eq. 8 concludes the method to evaluate algorithm quantitatively. 


$$
\left\{\begin{array}{l}
\omega_{1}=\frac{\mathrm{S}_{\mathrm{h}}}{\mathrm{S}_{\mathrm{h}}+\mathrm{S}_{\mathrm{l}}} \\
\omega_{2}=\frac{\mathrm{O}_{\mathrm{h}}}{\mathrm{O}_{\mathrm{h}}+\mathrm{O}_{1}}
\end{array} .\right.
$$

Among them, $\mathrm{S}_{\mathrm{h}}$ and $\mathrm{S}_{1}$ are pixel amount of shadow points correctly detected and mistakenly detected as object. Vice versa, the meaning of $\mathrm{O}_{\mathrm{h}}$ and $\mathrm{O}_{1}$ are obvious.

From the qualitative point of view, algorithm should be insensitive to noise, illumination, the color, texture and shape features of shadow, effective for different objects in different scenarios.

On the basis of evaluation standards above, detect shadows in 20 different scenarios with different objects inside. The average detection rate is shown in Table 1.

Table 1 result of qualitative evaluation

\begin{tabular}{|l|l|l|l|l|}
\hline & Indoor $\omega_{1 \%}$ & Indoor $\omega_{2 \%}$ & Outdoor $\omega_{1 \%}$ & Outdoor $\omega_{2 \%}$ \\
\hline Traditional method & 80.94 & 85.47 & 74.32 & 82.57 \\
\hline Proposed method & 86.95 & 90.64 & 80.83 & 86.93 \\
\hline
\end{tabular}

Algorithm proposed in this text has less calculation, is more accurate as shown in Table 1. Because it adopts an adaptive method of threshold decision which is more reasonable for processing different scenarios. This text uses the statistical feature of illumination difference to detect shadows, noise is not capable of affecting the statistical model a lot, thus the algorithm proposed is insensitive to noise, and shows better adaptability for different objects in different scenarios.

\section{Summary}

This text analyzes the illumination feature of shadows generated by moving objects in visible light image sequence according to Phong illumination model. Combine the foreground image and background image to calculate the brightness relative variation ratio as a way to describe illumination feature, on which through unique character shadow has could the threshold be found easily. Finally through experiments the effectiveness is proved, and compared with traditional algorithm, the proposed algorithm improves on performance and amount of calculation.

\section{References}

[1] ZHANG Shuifa, ZHANG Wensheng, et al. Background modeling and object detecting based on optical flow velocity field [J]. Journal of Image and Graphics, 2011, 16(2): 236-243.

[2] XUE Lixia, LUO Yanli, WANG Zuocheng. Detection algorithm of adaptive moving objects based on frame difference method [J]. Application Research of Computers, 2011, 28(4): 1551-1559.

[3] Kaewtrakulpong, P., Bowden, R.: An improved adaptive background mixture model for realtime tracking with shadow detection. In: Workshop on Advanced Video Based Surveillance Systems, pp.1 -5 (2001).

[4] Phong BT. Illuminationfor Computer Generated Pictures[ J]. Communication of ACM, 1975, 18( 6): 311-317.

[5] WU Liang, ZHOU Dongxiang, LIANG Hua, CAI Xuanping. A Shadow Detection Algorithm Based on Phong Lighting and Radiosity Model [J]. Modern Electronic Technology, 2008, 268(5):124-127. 